\title{
Inhibition of Neuropilin-1 Improves Non-Alcoholic Fatty Liver Disease via PI3K/AKT/Mtor Signaling in High-Fat- Diet induced Obese Mouse
}

Jian Zhou

wuhan puren hospital

Sihuan Xu

wuhan puren hospital

Yue Zhu

wuhan puren hospital

Xin Li

wuhan puren hospital

Ao Wang

wuhan puren hospital

Kun Liu

wuhan puren hospital

Junfang Hu

wuhan puren hospital

Li Li

wuhan Puren Hospital

Yan Liu ( $\sim$ liuyan@wust.edu.cn)

wuhan puren hospital https://orcid.org/0000-0002-9123-4974

\section{Research}

Keywords: Neuropilin-1, non-alcoholic fatty liver disease, Obesity, Inflammation, phosphatidylinositol 3-kinase, TOR Serine-Threonine Kinases

Posted Date: July 6th, 2021

DOl: https://doi.org/10.21203/rs.3.rs-665094/v1

License: (c) (i) This work is licensed under a Creative Commons Attribution 4.0 International License. Read Full License 


\section{Abstract}

Background: Research findings indicate Neuropilin-1 plays a critical role in lipid metabolism and obesity-associated insulin resistance, on such a basis, this study aims to explore the effects and working mechanism of Neuropilin-1 inhibition on the non-alcoholic fatty liver disease in high-fat-diet induced obese mice.

Methods: Firstly, the pcDNA3.1-NRP-1 recombinant plasmid containing Neuropilin-1 gene and the Neuropilin-1 gene RNA interference plasmid shRNA-NRP1 were successfully constructed. A total of 36 C57BL/ 6 mice were randomly assigned to 6 groups, blank group, control group, pcDNA3.1 injection group, pcDNA3.1-NRP-1 injection group, pGenesil-1.1 injection group and shRNA-NRP1 injection group. Expression of phospho-PI3K, phospho-AKT, phosphomTOR and Neuropilin-1 in liver was measured as well as body and liver weight, blood glucose, serum transaminases and lipid levels of the mice.

Results: The weight and liver mass of high-fat-diet fed mice injected with pcDNA3.1-NRP-1 were significantly higher than those from the control group, but their body weight and liver mass decreased significantly after shRNA-NRP1 injection. The results also showed that Neuropilin-1 expression can significantly influence the severity of hepatic steatosis in high-fat-diet fed mice, decreased serum FPG, LDL, AST, ALT levels and the expression of TNF- $\alpha$, IL-1 $\beta$, and IL-6 mRNA. In addition, the Neuropilin-1 expression will also influence the p-PI3K, p-AKT and p-mTOR in mice.

Conclusions: This study concluded that the inhibition of Neuropilin-1 could improve Non-alcoholic fatty liver disease by decreasing body weight and reduce inflammation in high-fat-diet induced obese mice by modulating the $\mathrm{PI} 3 \mathrm{~K} / \mathrm{AKT} / \mathrm{mTOR}$ signaling pathway.

\section{Background}

Non-alcoholic fatty liver disease (NAFLD), which is characterized by fat accumulation in hepatocytes [1], has aroused great concern in the world due to the high morbidity rate and its close link with hyperlipidemia, diabetes, and obesity [2]. NAFLD often occurs before hepatic steatosis. Simple steatosis is likely to occur during the occurrence of NAFLD and then develops into to nonalcoholic steatohepatitis (NASH) and liver cirrhosis at last $[3,4]$. During the formation and occurrence of NAFLD, obesity plays a critical role as a risk factor [5]. A high-fat-diet (HFD) may act as the 'first hit' leading to NAFLD while inflammation likely serves as a 'second hit' driving progression from NAFLD to NASH [6]. Currently, the treatment of NAFLD mainly aims at modifying risk factors, but there are no therapies directly targeting underlying liver pathology [7].

Neuropilins are units of transmembrane proteins whose molecular weight is around $130 \mathrm{kDa}$. One critical function of the neuropilins is to act as receptors and ensure the cell metabolism well-regulated. Neuropilin-1(NRP-1) and neuropilin-2 (NRP-2) which locates in 10q12 and 2q34 respectively encoded two proteins that belong to the neuropilin family too [8]. NRP-1 is also found on the surface of various kinds of cells, including the endothelium of blood [9] and lymphatic vessels [10], T-lymphocytes [11], nerve cells [12], glioma cells [13] and keratinocytes [14]. Research findings indicated the expression of neuropilins in several specific kinds of cancers, whether it's of epithelial or mesenchymal origin, including malignant melanoma, tumors of the gastrointestinal tract, etc $[15,16]$. NRP1 also exists in malignant gliomas, lung cancer and other kinds of malignant tumors $[17,18]$. Cao et al. showed that in two kinds of rat liver fibrosis models caused by hepatitis $C$ and fatty liver respectively, NRP-1 was highly expressed in the liver fibrosis tissue and was accompanied by the high expression of TGF- $\beta 1$ [19]. It was speculated that NRP-1 may promote the activation of hepatic stellate cells (HSC) and promote the occurrence of liver fibrosis by upregulating TGF- $\beta 1$. Further studies suggested that NRP1 may play an important role in TGF- $\beta 1$ and LAP-TGF- $\beta 1$ 
capture by cell membrane, thereby enhancing TGF- $\beta 1$ signal transduction and promoting TGF- $\beta 1$ secretion. Furthermore, promoted the epithelial-mesenchymal transition (EMT) [20, 21]. Recently, Dai et al. have revealed the new and crucial role that myeloid cell NRP1 might have played during the modulation of the insulin resistance associated with obesity [22]. In another research, Zhang et al. found that even though the mice were fed with a highfat diet, those lacked the genes encoding NRP1 and VEGFR1 did not consume lipids in a normal fat speed, and they excreted lipids instead, therefore, the mice rarely gained weight [23].

These research findings thus confirmed the critical role that NRP1 plays in lipid metabolism and insulin resistance that associated with obesity, however, there's still no report covering the correlation between NRP1 and HFD-induced NAFLD. PI3K/Akt/mTOR signaling pathway plays an important role in the occurrence and development of liver fibrosis [24]. Activated PI3K/Akt/mTOR promoted the formation and development of liver fibrosis by regulating the proliferation and migration of hepatic stellate cells (HSC), increasing the expression of Collagen $\nabla$ and affecting the balance of MMP and TIMP [25]. Therefore, blocking PI3K/Akt/mTOR signaling pathway can induce the apoptosis of HSC, inhibit the secretion of ECM and promote the degradation of ECM, thus delaying the progress of liver fibrosis. Both NRP-1 and PI3K/Akt/mTOR signal transduction pathways have effects on hepatic stellate cells (HSC), and further promote the occurrence of liver fibrosis. However, there has been no research on the correlation between NRP-1 and PI3K/Akt/mTOR signal transduction pathways. However, there is no study showed the correlations between NRP-1 and PI3K/Akt/mTOR signal transduction pathways, thus, this study was designed to investigate the effect of NRP1 on HFD-induced NAFLD and to explore the underlying mechanism via PI3K/AKT/mTOR signaling.

\section{Materials And Methods}

\section{Bacteria, carriers and experimental reagents}

pGenesil-1.1, pCR-blunt, pcDNA3.1, DH5a strains were from the laboratory of research center; SYBR Premix EX Taq, T4 DNA ligase, Taq DNA polymerase were purchased from TaKaRa; Ecoll, Hind III, Sacl endonucleases were purchased from NEB Company; RNeasy kit, plasmid extraction kit and gel recovery kit were purchased from GIAGEN; Trizol, liposome Lipofectamine TM2000 transfection kit was purchased from Invitrogen; P38, p-P38, JNK, p-JNK, NRP-1 antibodies were purchased from Cell Signaling Technology; NRP1 interference sequence synthesis, primer synthesis and gene sequencing were performed by Shanghai Shenggong Bioengineering Technology Service Co., Ltd.

\section{Construction and identification of recombinant plasmid pcDNA3.1-NRP-1}

\section{Construction of pCR-blunt-NRP-1 cloning vector}

Total RNA of mouse liver tissue was extracted by TRIzol method, NRP-1 gene was amplified by reverse transcription, PCR product was subjected to agarose gel electrophoresis, and the target gene was recovered. Fragments of the target gene were ligated with the pCR-blunt vector by T4 DNA ligase, and then the product transformed competent cells, the monoclonal colony was picked overnight. The small amount of the extracted plasmid was identified by Ecoll single digestion and sequenced. After sequencing, the plasmid was correctly sequenced and named pCR-bluntNRP-1.

\section{Construction and identification of pcDNA3.1-NRP-1 expression vector}


The recombinant plasmids pCR-blunt-NRP-1 and pcDNA3.1 were digested with Ecoll, and the reaction product was detected by $0.7 \%$ agarose gel electrophoresis and the target band was observed. The GRP-recovered NRP-1 gene fragment was ligated with pcDNA3.1, T4DNA ligase. The ligation product then transformed competent cell DH-5a. On the second day, several monoclonal colonies were picked, of which the plasmid was extracted after shaking the bacteria and placed its overnight, and the recombinant plasmid was identified by single digestion with Hind III. The correctly digested plasmid was named pcDNA3.1-NRP-1, and the bacterial solution was stored at $-80^{\circ} \mathrm{C}$.

\section{Construction of NRP1 gene RNA interference plasmid of shRNA- NRP1}

An interference sequence of 19 nt was designed based on the NRP1 gene mRNA sequence: NRP1: GGACCCATACCAGAGAATT (target 706 bp); NRP-1A: 5'CACCGGACCCATACCAGAGAATTCTATGGACAAATTCTCTGGTATGGGT CCTTTTTTG-3', NRP-1B: 5'AGCTCAAAAAAGGACCCATACCAGAGAATTT GTCCATAGAATTCTCTGGTATGGGTCC-3', primer structure: CACC + Sense + Loop + Antisense + termination signal + Sacl. The sense strand was anneal-linked to the single strand of the antisense oligonucleotide, and the double-stranded oligonucleotide was ligated to the pGenesil-1.1 linear vector (containing the hU6 promoter) by T4 DNA ligase, and the ligated product was transformed into E. coli DH5a, and inoculated the incubated overnight at $37^{\circ} \mathrm{C}$ on kanamycin resistant LB plates. Monoclonal colonies were picked, inoculated into kanamycin-resistant LB medium, and cultured overnight at $37^{\circ} \mathrm{C}$ in a constant temperature shaker. The plasmid was extracted and identified by Sacl digestion. The multiple cloning sites (MCS) of the plasmid pGenesil-1.1 were as follows: Mlul-hU6promoter- InsertDNA-Sacl, a Sacl cleavage site was designed in the fragment of inserted target gene, and the plasmid pGenesil-1.1 itself had a Sacl. The cleavage site, if inserted correctly, the plasmid can be cleaved by Sacl to a 916 bp DNA band. Enzyme digestion was performed to identify the correct plasmid sequencing.

\section{Experimental design}

A total of 36 male C57BL/ 6 mice ( 6 weeks old, $18.44 \pm 0.92 \mathrm{~g}$ ) were purchased from experimental animal center of Tongji Medical College affiliated to Huazhong University of Science and Technology. The mice were housed in a clean room maintained at $24 \pm 2^{\circ} \mathrm{C}$ in a $12 \mathrm{~h}$ : $12 \mathrm{~h}$ light: dark cycle, with free access to food and water. All animal experiments were approved by the Animal Care and Use Committee of Tianyou Hospital affiliated to Wuhan University of Science and Technology with the Ethics Protocol number TY20180212. Mice were randomly assigned to 6 groups. Group A $(n=6)$ were fed with regular diet $(R D)$ for 16 weeks, and the other groups were fed with HFD for 16 weeks. After 8 weeks, Group B $(n=6)$ received $100 \mathrm{ug} / \mathrm{kg}$ physiological saline by intravenous injection, Group C $(n=6)$ received $100 \mathrm{ug} / \mathrm{kg}$ pcDNA3.1 by intravenous injection, Group D $(n=6)$ received $100 \mathrm{ug} / \mathrm{kg}$ pcDNA3.1-NRP-1 by intravenous injection, Group E $(n=6)$ received $100 \mathrm{ug} / \mathrm{kg}$ pGenesil-1.1 by intravenous injection, Group $F(n=6)$ received $100 \mathrm{ug} / \mathrm{kg}$ shRNA-NRP1 by intravenous injection. All the groups received 2 injections in a week (on Monday and Friday respectively) (Table 1). HFD and RD came from Meidisen Biomedical Company (Jiangsu, China). The diets for mice in the experiment were in accordance with AIN93 recommendations, of which $60 \%$ calories of the high-fat diet (research diet D12492) came from fat, $20 \%$ from proteins and the rest $20 \%$ from carbohydrates. Of the regular diet (research diet D12450B), 10\% calories came from fat, 20\% from proteins and 70\% from carbohydrates. In the end of the experiment, mice were anaesthetized with pentobarbital sodium, livers were removed and blood was collected via cardiac puncture.

\section{Evaluation of body and liver weight}


Body weight of the mice was measured every 2 weeks consistently between $7 \mathrm{pm}$ and 8 pm, liver weight was measured at the end of the experiment after sacrifice.

\section{Levels of blood transaminase and lipid}

The serum levels of alanine aminotransferase (ALT) and aspartate aminotransferase (AST), triglycerides (TG), lowdensity cholesterol (LDL-C) and high-density cholesterol (HDL-C) were measured spectrophotometrically in accordance with the instructions (NJJCBIO, Jiangsu, China).

\section{Histological analyses}

Took a representative mouse from each group, then took the liver and epididymal adipose tissue of the mice and fixed them in $10 \%$ buffered formalin, embedded in paraffin, cut into thick sections of $8 \mu \mathrm{m}$. Stained with hematoxylin and eosin for histological examination of lipid droplets, and then took an Olympus SZX10 microscope (Tokyo, Japan) to get the images. The software Image Pro Plus 7.01 (Media Cybernetics, Silver Springs, MD, USA) was taken to analyze the volume density ( $\mathrm{Vv})$ of hepatic steatosis, and the results are listed as below [26].

\section{Real-time reverse-transcriptase polymerase chain reaction (qRT- PCR)}

Trizol was taken to isolate the total mRNA of mouse liver. According to the instruction, two micrograms of total RNA were used for cDNA synthesis with the help of the RNase kit. 7900HT real-time PCR system (ABI, CA, USA) was taken to perform a quantitative real-time SYBR Green quantitative RT-PCR, and the expression of target genes was determined. Glyceraldehyde 3-phosphate dehydrogenase (GAPDH) mRNA was taken as an endogenous control and the $\Delta \Delta \mathrm{Ct}$ method was used for the analysis of relevant data. Primer sequences are provided in Table 2.

\section{Western blotting}

Expression of phospho-PI3K (p-PI3K), phospho-AKT (p-AKT), phospho-mTOR (p-mTOR), PI3K, AKT, mTOR and NRP1 in liver were measured by Western blotting as described [27]. Proteins were separated on 12.5\% SDS-PAGE gels and transferred onto nitrocellulose membranes. The expression of p-PI3K, p-AKT, p-mTOR, PI3K, AKT, mTOR and NRP-1 were analyzed with a rabbit monoclonal antibody (1: 1000) primary antibody, and a rabbit-antimouse IgGHRP (1:1000) secondary antibody. GAPDH (1: 1000 dilutions for the primary antibody and 1: 1000 dilutions for the secondary antibody) was used as an endogenous control. Western blot bands were scanned and quantified by densitometry analysis using an image analyzer Quantity One System (Odyssey, USA).

\section{Statistical evaluation}

Statistical analyses were performed using software of SPSS 21.0 (Chicago, IL) and GraphPad Prism (GraphPad Software Inc). Data were presented as mean values \pm standard errors. The one-way ANOVA test was used to determine differences between groups by comparing the internal variability of the control groups with the variability among all experimental groups. Multiple comparisons between the groups were performed using Student-NewmanKeuls method as a post hoc test. $P<0.05$ was considered statistically significant. All assays were performed in triplicate.

Results

\section{NRP1 mRNA}


As shown in Fig. 1, the expression of NRP1 mRNA in liver tissue of group D was significantly higher than that of other groups $(P<0.05)$. The expression of NRP1 mRNA in liver tissues of mice in group $A, B, C$ and $E$ was significantly higher than that in group $\mathrm{F}(P<0.05)$, but there was no significant difference in the expression of NRP1 mRNA in liver tissues of mice in group $A, B, C$ and $E(P>0.05)$.

\section{NRP1 protein}

As shown in Fig. 2A and Fig. 2B, the expression of NRP1 protein in liver tissue of mouse from group $D$ was significantly higher than that of mouse from other groups $(P<0.05)$. The expression of NRP1 protein in liver tissues of mouse in group $A, B, C$ and $E$ was significantly higher than that of mouse from group $F$. There was no significant difference between the expression of NRP1 protein in liver tissues of mouse from group $A, B, C$ and $E(P>0.05)$.

\section{Body weight and liver mass}

As shown in Fig. 3A, at week 8, the weight of mouse in group A was significantly lower than that in other groups $(P<$ 0.05). The weight of mouse in group $D$ increased significantly after a tail injection of pcDNA3.1-NRP-1, and their body weight was significantly higher than that of the mouse from Groups $\mathrm{B}, \mathrm{C}$, and $\mathrm{E}$ at all time points $(P<0.05)$. The body weight of mouse from Group $F$ decreased significantly after shRNA-NRP1 injection in the tail vein, and the body weight of mouse in this group was significantly lower than that of mouse from Group B, C, E at each time point $(P<0.05)$, but the body weight of mouse from Group $B, C$, and $E$ groups was not significantly different at any time point $(P>0.05)$. As shown in Fig. 3B, the liver mass of mouse from Group $D$ was significantly higher than that of mouse from other groups $(P<0.05)$, and the liver mass of mouse from Group $\mathrm{B}, \mathrm{C}$ and $\mathrm{E}$ was not significantly different $(P>0.05)$, but was significantly higher than that of mouse from Group $\mathrm{A}$ and $\mathrm{F}(P<0.05)$, the liver mass of mouse from Group A and F was not statistically significant $(P>0.05)$.

\section{Glucose and lipid metabolism}

As shown in Table 3, the serum levels of FPG, LDL, AST and ALT of mouse in group D were significantly higher than those of mouse in other groups $(P<0.05)$. The serum levels of FPG, LDL and AST of mouse in group $B, C$ and $E$ were similar. The levels of ALT and ALT of mouse from groups B, C, D, E were not significantly different $(P>0.05)$, but they were significantly higher than those of mouse in group $A$ and $F(P<0.05)$. There was no statistical difference in serum FPG, LDL, AST and ALT levels between mouse in Group A and Group F $(P>0.05)$.

\section{Histological analyses of liver}

As shown in Fig. 4A-D, the liver steatosis ratio and adipocyte diameter of mouse in Group D were significantly higher than those of mouse in other groups $(P<0.05)$. There was no significant difference in liver steatosis ratio and adipocyte diameter between mouse in Group $B, C$ and $\mathrm{E}$. $(P>0.05)$, but the levels were significantly higher than those of mouse in Group A and $\mathrm{F}(P<0.05)$, and there was no significant difference in liver steatosis ratio and adipocyte diameter between mouse in Group $A$ and $\mathrm{F}(P>0.05)$.

\section{Quantification of pro-inflammatory cytokines}

As shown in Fig. 5, the mRNA levels of TNF-a, IL-1 $\beta$ and IL-6 in the liver tissues of mouse in Group D were significantly higher than those of mouse in other groups $(P<0.05)$. The levels of TNF-a, IL-1 $\beta$ and IL- 6 mRNA of liver tissue of mouse in Group B, C and E was no significantly different $(P>0.05)$, but they were significantly higher than 
those of mouse in Group A and $F(P<0.05)$. The levels of TNF-a, IL-1 $\beta$ and IL-6 mRNA of liver tissue of mouse in Group A and Group F were not significantly different $(P>0.05)$, and the levels of IL-10, JNK and IKK- $\beta$ mRNA of liver tissue of mouse in all groups were not significantly different $(P>0.05)$.

\section{The related proteins expression of PI3K/AKT/mTOR signaling pathway}

As shown in Fig. 6A and Fig. 6B, There were no significant differences in PI3K, AKT, mTOR protein levels between the liver tissues of mouse in all groups $(P>0.05)$. There were no significant differences in $\mathrm{p}-\mathrm{PI} 3 \mathrm{~K}, \mathrm{p}-\mathrm{AKT}, \mathrm{p}-\mathrm{mTOR}$ protein levels between the liver tissues of mouse in Group $\mathrm{B}, \mathrm{C}, \mathrm{E}(P>0.05)$, but they were significantly higher than those of mouse in Group $A$ and $F(P<0.05)$, and lower than those of mouse in Group $\mathrm{D}(P<0.05)$. There were no significant differences in p-PI3K,p-AKT, p-mTOR protein levels between the liver tissues of mouse in Group $A$ and $\mathrm{F}(P>0.05)$. The phosphor/total ratio of each protein were shown in Fig. 6C.

\section{Discussion}

NRP-1 is an important member of the NRP family and plays an important role in neurodevelopment, angiogenesis, tumor invasion, metastasis and immunity [28-31]. NRP-1 initially has been identified as a coreceptor for Sema3 and growth factors, including vascular endothelial growth factor, transforming growth factor-b, hepatocyte growth factor and platelet-derived growth factor [32-35], and it have been shown to be strongly expressed in immune cells

and to regulate immune response [36,37]. In macrophages, ablation of NRP1 results in decreased tumor growth and metastasis via enhanced infifiltration of tumor-associated macrophages into normoxic tumor regions, which abolishes the proangiogenic and immunosuppressive functions of tumor-associated macrophages [38]. Consistently, Miyauchi et al. reported that NRP1 ablation in glioma-associated microglia and macrophages suppresses glioma progression by promoting M1 macrophage polarization [39]. Recent findings further established the involvement of the Sema3A/Nrp1 axis, as well as M1/M2 macrophages, with respect to tumorigenic processes [40]. However, specific functions of NRP1 in the context of metabolic dysfunction, such as NAFLD, have not been investigated yet. Studies have shown that: NRP1 may play an important role in the process of capturing TGF- $\beta 1$ and LAP-TGF- $\beta 1$ by cell membrane, thereby enhancing TGF- $\beta 1$ signal transduction and promoting TGF- $\beta 1$ secretion, thus promoting the occurrence and development of EMT [21]. EMT-producing cells may enhance cell hardness and provide fibrotic cytokines. Indirect stimulation of myofibroblasts induces fibrosis [41]. Other studies have shown that NRP-1 plays a novel role in tumor progression by enhancing the autorine HGF/ c-Met pathway, suggesting that NRP1 may also play a role as a functional receptor for $\operatorname{HGF}[42,43]$. HGF regulates the survival, proliferation and migration of endothelial cells, matrix deposition and degradation, and the formation of capillary-like structures through c-Met [44]. All the above studies suggest that NRP-1 can influence fibrosis, but its role in HE has not been reported in the literature.

This study is to observe the effect of NRP-1 expression enhancement and inhibition of NRP-1 expression on the formation of HFD-induced obese mouse NAFLD, and to explore its mechanism of action. Long-term high fat diet feeding in mice could cause whole-body energy imbalance, resulting in a series of obesity-associated metabolic disorders. To study the relation about NRP-1 and NAFLD, we established a NAFLD model. The results suggested that elevated NRP-1 expression can significantly increase the body weight and liver mass of NAFLD model mouse, while inhibition of NRP-1 expression can significantly reduce the body weight and liver mass of NAFLD model mouse. 
The results also suggest an increase in NRP-1 expression can aggravate the abnormality of blood lipid and liver function of NAFLD model mice, and inhibiting the expression of NRP-1 can significantly alleviate the abnormality of blood lipid and liver function of NAFLD model mouse. In addition, H\&E staining results also indicated that the increase of NRP-1 expression may aggravate the degree of liver degeneration of NAFLD model mouse and inhibit the expression of NRP-1. The degree of NAFLD model mouse liver degeneration can be significantly reduced.

Insulin resistance is considered to be the central link in the development of NAFLD and metabolic syndrome. Inflammatory factors affect insulin sensitivity, and inflammatory factors are involved in the development and progression of NAFLD, adipose tissue and liver immune dysfunction in patients with NAFLD, abnormal macrophages. Macrophages cells are important sources of inflammatory factors, and both fat and hepatocytes secrete inflammatory factors such as TNF-a and IL helps to mediate macrophage infiltration $[45,46]$. To explore the possible mechanism of NRP1's effects on NAFLD, we measured mRNA levels of pro-inflammatory makers. Then we found that the inhibition of NRP1 expression significantly reduced the mRNA level of pro-inflammatory cytokines including TNF- $a$, IL-1 $\beta$, and IL-6, which were increased in HFD fed mouse, but the enhanced NRP1 expression significantly elevated the mRNA level of pro-inflammatory cytokines.

NAFLD is currently considered to be a metabolic stress liver injury closely related to insulin resistance and genetic susceptibility, in which insulin resistance; oxidative stress and inflammatory response play an important role in the development of NAFLD [47-49]. As a key pathway of insulin signaling, PI3K/Akt/mTOR participates in glycolipid metabolism by regulating insulin level [50]. However, PI3K/Akt/mTOR signal transduction disorders caused by various factors can cause insulin resistance (IR), promote the occurrence and development of NAFLD [51], and also participate in the pathophysiological process of oxidative damage of cells [52]. Phosphatidylinositol 3kinase/protein kinase B/mammalian target of rapamycin (PI3K/AKT/mTOR) was an important signal transduction pathway in cells, which was closely related to cell proliferation, apoptosis, angiogenesis and other biological behaviors [53]. PI3K was an important member of the superfamily of growth factor receptor signal transduction process. AKT and mTOR were important downstream genes of PI3K, and PI3K could affect families related to cell apoptosis through AKT, mTOR and effector molecules, thereby affecting cell apoptosis [54]. Inflammation were a major factor in the occurrence and development of chronic liver disease and lead to liver fibrosis and cirrhosis, PI3K and AKT could adjust the key inflammatory cytokine activation of immune cells [55], PI3K and mTOR limited the activation of immune cells, adjusted in the liver inflammation and fibrosis formation, regulated the proliferation and migration of HSC in the process of liver fibrosis, synthesis and degradation of the ECM by raising the key antiinflammatory cytokine interleukin-10 and inhibiting proinflammatory cytokines [56]. In addition, in the state of inflammation, the PI3K/AKT/mTOR signaling pathway was over-activated, resulted in excessive transcription of downstream target genes, abnormal cell proliferation, tissue repair disorders, and aggravating tissue damage. Based on the consensus that "IR $\rightarrow$ abnormal lipid metabolism $\rightarrow$ liver fat deposition $\rightarrow$ oxygen stress and lipid peroxidation $\rightarrow$ NAFLD changes", the PI3K/AKT/mTOR signaling pathway was expected to be the target for improving NAFLD. To explore the possible mechanism of NRP1's effects on NAFLD, we measured the active levels of PI3K/AKT/mTOR signaling. The expression of PI3K, AKT and mTOR were similar in six groups, but the phosphorylated levels of PI3K, AKT and mTOR increased in HFD fed mouse when compared with that of RD fed mouse. The activation of the PI3K/AKT/mTOR signaling molecules by HFD was reduced by the regulation of NRP1 expression, but elevated through an enhance NRP1 expression. HFD leads to inflammation and weight gain, and the inhibition of NRP1 expression could alleviate the inflammation as well as obesity caused by HFD. Taken together, our data indicate that NRP1 affects the PI3K/AKT/mTOR signaling pathways leading to elevated inflammatory responses induced by HFD. 
There are still some limitations in this study. Only the effects of enhancement or inhibition of NRP1 expression on proteins related to $\mathrm{PI} 3 \mathrm{~K} / \mathrm{Akt} / \mathrm{mTOR}$ signal transduction pathway were studied, and it was concluded that NRP1 may have an effect on non-alcoholic fatty liver disease by regulating PI3K/Akt/mTOR signal transduction pathway. Further investigation is needed through the use of PI3K/Akt/mTOR signaling pathway agonists or inhibitors for interference. In addition, does NRP1 regulate other signaling pathways besides PI3K/Akt/mTOR signaling pathway to exert influence on non-alcoholic fatty liver disease? This will require further study.

\section{Conclusions}

This study concluded that HFD leads to inflammation and weight gain, and the inhibition of NRP1 expression could alleviate the inflammation as well as obesity caused by HFD. Taken together, our data indicate that NRP1 affects the PI3K/AKT/mTOR signaling pathways leading to elevated inflammatory responses induced by HFD.

\section{Declarations}

\section{Ethics approval and consent to participate}

Not applicable.

\section{Consent for publication}

Not applicable.

\section{Availability of data and materials}

The datasets used and/or analysed during the current study are available from the corresponding author on reasonable request.

\section{Competing interests}

The authors declare that they have no competing interests.

\section{Funding}

None.

\section{Authors' contributions}

Jian Zhou: Conceptualization, Methodology, Data curation, Investigation, Validation, Writing - original draft. Sihuan Xu: Data curation, Investigation,Visualization, Writing - review \& editing. Yan Liu: Investigation, Formal analysis, Validation, Writing - review \& editing, Supervision. Yue Zhu: Validation, Investigation. Xin Li: Validation, Investigation. Ao Wang: Data curation, Investigation. Li Li: Data curation, Investigation. Kun Liu: Validation, Investigation. Junfang Hu: Validation, Investigation.

\section{Acknowledgments}

None.

\section{References}


1. Sporea I, Popescu A, Dumitrașcu D, Brisc C, Nedelcu L, Trifan A, et al. Nonalcoholic Fatty Liver Disease: Status Quo. J Gastrointestin Liver Dis. 2018;27(4):439-48.

2. Younossi ZM. Non-alcoholic fatty liver disease - A global public health perspective. J Hepatol. 2019;70(3):53144.

3. Tanaka N, Kimura T, Fujimori N, Nagaya T, Komatsu M, Tanaka E. Current status, problems, and perspectives of non-alcoholic fatty liver disease research. World J Gastroenterol. 2019;25(2):163-77.

4. Wong SW, Ting YW, Chan WK. Epidemiology of non-alcoholic fatty liver disease-related hepatocellular carcinoma and its implications. JGH Open. 2018;2(5):235-41.

5. Lau LHS, Wong SH. Microbiota. Obesity and NAFLD. Adv Exp Med Biol. 2018;1061:111-25.

6. Ullah R, Rauf N, Nabi G, Ullah H, Shen Y, Zhou YD, et al. Role of Nutrition in the Pathogenesis and Prevention of Non-alcoholic Fatty Liver Disease: Recent Updates. Int J Biol Sci. 2019;15(2):265-76.

7. Thanapirom K, Tsochatzis EA. Non-alcoholic fatty liver disease (NAFLD) and the quest for effective treatments. Hepatobiliary Surg Nutr. 2019;8(1):77-9.

8. Pellet-Many C, Frankel P, Jia H, Zachary I. Neuropilins: structure, function and role in disease. Biochem J. 2008;411(2):211-26.

9. Wang Y, Wang E, Zhang Y, Madamsetty VS, Ji B, Radisky DC, et al. Neuropilin-1 maintains dimethylarginine dimethylaminohydrolase 1 expression in endothelial cells, and contributes to protection from angiotensin IIinduced hypertension. Faseb j. 2019;33(1):494-500.

10. Ochsenbein AM, Karaman S, Jurisic G, Detmar M. The role of neuropilin-1/semaphorin $3 A$ signaling in lymphatic vessel development and maturation. Adv Anat Embryol Cell Biol. 2014;214:143-52.

11. Gao YL, Chai YF, Qi AL, Yao Y, Liu YC, Dong N, et al. Neuropilin-1highCD $4^{\llbracket C D} 25^{\square}$ Regulatory T Cells Exhibit Primary Negative Immunoregulation in Sepsis. Mediators Inflamm. 2016;2016:7132158.

12. Erskine L, François U, Denti L, Joyce A, Tillo M, Bruce F, et al. VEGF-A and neuropilin 1 (NRP1) shape axon projections in the developing CNS via dual roles in neurons and blood vessels. Development. 2017;144(13):2504-16.

13. Chen L, Miao W, Tang X, Zhang H, Wang S, Luo F, et al. The expression and significance of neuropilin-1 (NRP-1) on glioma cell lines and glioma tissues. J Biomed Nanotechnol. 2013;9(4):559-63.

14. Riese A, Eilert Y, Meyer Y, Arin M, Baron JM, Eming S, et al. Epidermal expression of neuropilin 1 protects murine keratinocytes from UVB-induced apoptosis. PLoS One. 2012;7(12):e50944.

15. Shahrabi-Farahani S, Wang L, Zwaans BM, Santana JM, Shimizu A, Takashima S, et al. Neuropilin 1 expression correlates with differentiation status of epidermal cells and cutaneous squamous cell carcinomas. Lab Invest. 2014;94(7):752-65.

16. Matkar PN, Jong ED, Ariyagunarajah R, Prud'homme GJ, Singh KK, Leong-Poi H. Jack of many trades: Multifaceted role of neuropilins in pancreatic cancer. Cancer Med. 2018;7(10):5036-46.

17. Hu C, Zhu P, Xia Y, Hui K, Wang M, Jiang X. Role of the NRP-1-mediated VEGFR2-independent pathway on radiation sensitivity of non-small cell lung cancer cells. J Cancer Res Clin Oncol. 2018;144(7):1329-37.

18. Caponegro MD, Moffitt RA, Tsirka SE. Expression of neuropilin-1 is linked to glioma associated microglia and macrophages and correlates with unfavorable prognosis in high grade gliomas. Oncotarget. 2018;9(86):35655-65.

19. Cao S, Yaqoob U, Das A, Shergill U, Jagavelu K, Huebert RC, et al. Neuropilin-1 promotes cirrhosis of the rodent and human liver by enhancing PDGF/TGF-beta signaling in hepatic stellate cells. J Clin Investig. 
2010;120(7):2379-94.

20. Hu B, Guo P, Bar-Joseph I, Imanishi Y, Jarzynka MJ, Bogler O, et al. Neuropilin-1 promotes human glioma progression through potentiating the activity of the HGF/SF autocrine pathway. Oncogene. 2007;26(38):557786.

21. Matsushita A, Götze T, Korc M. Hepatocyte growth factor-mediated cell invasion in pancreatic cancer cells is dependent on neuropilin-1. Cancer research. 2007;67(21):10309-16.

22. Dai X, Okon I, Zou MH. Myeloid cell neuropilin 1 ameliorates high-fat diet-induced insulin resistance via suppression of Nlrp3 inflammasome. Macrophage (Houst). 2017;4.

23. Zhang F, Zarkada G, Han J, Li J, Dubrac A, Ola R, et al. Lacteal junction zippering protects against diet-induced obesity. Science. 2018;361(6402):599-603.

24. Birchmeier C, Birchmeier W, Gherardi E, Vande Woude GF. Met, metastasis, motility and more. Nature reviews Molecular cell biology. 2003;4(12):915-25.

25. Zeng L, Tang WJ, Yin JJ, Zhou BJ. Signal transductions and nonalcoholic fatty liver: a mini-review. Int J Clin Exp Med. 2014;7(7):1624-31.

26. Catta-Preta M, Mendonca LS, Fraulob-Aquino J, Aguila MB, Mandarim-de-Lacerda CA. A critical analysis of three quantitative methods of assessment of hepatic steatosis in liver biopsies. Virchows Arch. 2011;459(5):477-85.

27. Gusdon AM, Fernandez-Bueno GA, Wohlgemuth S, Fernandez J, Chen J, Mathews CE. Respiration and substrate transport rates as well as reactive oxygen species production distinguish mitochondria from brain and liver. BMC Biochem. 2015;16:22.

28. Matsuda S, Kobayashi M, Kitagishi Y. Roles for PI3K/AKT/PTEN Pathway in Cell Signaling of Nonalcoholic Fatty Liver Disease. ISRN endocrinology. 2013;2013:472432.

29. Yu JS, Cui W. Proliferation, survival and metabolism: the role of PI3K/AKT/mTOR signalling in pluripotency and cell fate determination. Development. 2016;143(17):3050-60.

30. Niland S, Eble JA. Neuropilins in the Context of Tumor Vasculature. International journal of molecular sciences. 2019;20(3).

31. Dong JC, Cheng GH, Shan YX, Wu N, Shao ML, Li PW, et al. Role of PLC-PIP2 and CAMP-PKA signal pathways in radiation-induced immune-suppressing effect. Biomed Environ Sci: BES. 2014;27(1):27-34.

32. He Z, Tessier-Lavigne M. Neuropilin is a receptor for the axonal chemorepellent Semaphorin III. Cell. 1997;90(4):739-51.

33. Kolodkin AL, Levengood DV, Rowe EG, Tai YT, Giger RJ, Ginty DD. Neuropilin is a semaphorin III receptor. Cell. 1997;90(4):753-62.

34. Soker S, Takashima S, Miao HQ, Neufeld G, Klagsbrun M. Neuropilin-1 is expressed by endothelial and tumor cells as an isoform-specific receptor for vascular endothelial growth factor. Cell. 1998;92(6):735-45.

35. Kumanogoh A, Kikutani H. Immunological functions of the neuropilins and plexins as receptors for semaphorins. Nat Rev Immunol. 2013;13(11):802-14.

36. Tordjman R, Lepelletier Y, Lemarchandel V, Cambot M, Gaulard P, Hermine O, et al. A neuronal receptor, neuropilin-1, is essential for the initiation of the primary immune response. Nat Immunol. 2002;3(5):477-82.

37. Bruder D, Probst-Kepper M, Westendorf AM, Geffers R, Beissert S, Loser K, et al. Neuropilin-1: a surface marker of regulatory T cells. Eur J Immunol. 2004;34(3):623-30. 
38. Delgoffe GM, Woo SR, Turnis ME, Gravano DM, Guy C, Overacre AE, et al. Stability and function of regulatory T cells is maintained by a neuropilin-1-semaphorin-4a axis. Nature. 2013;501(7466):252-6.

39. Casazza A, Laoui D, Wenes M, Rizzolio S, Bassani N, Mambretti M, et al. Impeding macrophage entry into hypoxic tumor areas by Sema3A/Nrp1 signaling blockade inhibits angiogenesis and restores antitumor immunity. Cancer Cell. 2013;24(6):695-709.

40. Miyauchi JT, Chen D, Choi M, Nissen JC, Shroyer KR, Djordevic S, et al. Ablation of Neuropilin 1 from gliomaassociated microglia and macrophages slows tumor progression. Oncotarget. 2016;7(9):9801-14.

41. Wang J, Chu ES, Chen HY, Man K, Go MY, Huang XR, et al. microRNA-29b prevents liver fibrosis by attenuating hepatic stellate cell activation and inducing apoptosis through targeting PI3K/AKT pathway. Oncotarget. 2015;6(9):7325-38.

42. Wei L, Chen Q, Guo A, Fan J, Wang R, Zhang H. Asiatic acid attenuates CCl(4)-induced liver fibrosis in rats by regulating the PI3K/AKT/mTOR and Bcl-2/Bax signaling pathways. Int Immunopharmacol. 2018;60:1-8.

43. Peng X, Moore MW, Peng H, Sun H, Gan Y, Homer RJ, et al. CD4 + CD25 + FoxP3 + Regulatory Tregs inhibit fibrocyte recruitment and fibrosis via suppression of FGF-9 production in the TGF- $\beta 1$ exposed murine lung. Front Pharmacol. 2014;5:80.

44. Valdembri D, Regano D, Maione F, Giraudo E, Serini G. Class 3 semaphorins in cardiovascular development. Cell adhesion migration. 2016;10(6):641-51.

45. Ipsen DH, Lykkesfeldt J, Tveden-Nyborg P. Molecular mechanisms of hepatic lipid accumulation in nonalcoholic fatty liver disease. Cell Mol Life Sci. 2018;75(18):3313-27.

46. Takahashi Y, Sugimoto K, Inui H, Fukusato T. Current pharmacological therapies for nonalcoholic fatty liver disease/nonalcoholic steatohepatitis. World J Gastroenterol. 2015;21(13):3777-85.

47. Chávez-Talavera O, Haas J, Grzych G, Tailleux A, Staels B. Bile acid alterations in nonalcoholic fatty liver disease, obesity, insulin resistance and type 2 diabetes: what do the human studies tell? Curr Opin Lipidol. 2019;30(3):244-54.

48. Palladini G, Di Pasqua LG, Berardo C, Siciliano V, Richelmi P, Mannucci B, et al. Fatty Acid Desaturase Involvement in Non-Alcoholic Fatty Liver Disease Rat Models: Oxidative Stress Versus Metalloproteinases. Nutrients. 2019;11(4).

49. Farrell GC, Haczeyni F, Chitturi S. Pathogenesis of NASH: How Metabolic Complications of Overnutrition Favour Lipotoxicity and Pro-Inflammatory Fatty Liver Disease. Adv Exp Med Biol. 2018;1061:19-44.

50. Roy S, Bag AK, Singh RK, Talmadge JE, Batra SK, Datta K. Multifaceted Role of Neuropilins in the Immune System: Potential Targets for Immunotherapy. Frontiers in immunology. 2017;8:1228.

51. Kazemi M, Carrer A, Moimas S, Zandonà L, Bussani R, Casagranda B, et al. VEGF121 and VEGF165 differentially promote vessel maturation and tumor growth in mice and humans. Cancer Gene Ther. 2016;23(5):125-32.

52. Gabasa M, Duch P, Jorba I, Giménez A, Lugo R, Pavelescu I, et al. Epithelial contribution to the profibrotic stiff microenvironment and myofibroblast population in lung fibrosis. Molecular biology of the cell. 2017;28(26):3741-55.

53. Yang L, Liu Y, Wang M, Qian Y, Dai X, Zhu Y, et al. Celastrus orbiculatus extract triggers apoptosis and autophagy via PI3K/Akt/mTOR inhibition in human colorectal cancer cells. Oncol Lett. 2016;12(5):3771-8.

54. Wang H, Zhang C, Xu L, Zang K, Ning Z, Jiang F, et al. Bufalin suppresses hepatocellular carcinoma invasion and metastasis by targeting HIF-1a via the PI3K/AKT/mTOR pathway. Oncotarget. 2016;7(15):20193-208.

Page $12 / 18$ 
55. Pettinelli P, Obregón AM, Videla LA. Molecular mechanisms of steatosis in nonalcoholic fatty liver disease. Nutr Hosp. 2011;26(3):441-50.

56. Weichhart T, Säemann MD. The PI3K/Akt/mTOR pathway in innate immune cells: emerging therapeutic applications. Ann Rheum Dis. 2008;67(Suppl 3):iii70-4.

\section{Tables}

Table 1. Treatment of different groups

\begin{tabular}{|ll|}
\hline Group & Methods \\
A & fed with regular diet $(\mathrm{RD})$ by hydrodynamie injection \\
B & HFD+physiological saline $(100 \mathrm{ug} / \mathrm{kg})$ by hydrodynamie injection \\
C & HFD+pcDNA3.1 $(100 \mathrm{ug} / \mathrm{kg})$ by hydrodynamie injection \\
D & HFD+pcDNA3.1-NRP-1(100 ug/ kg) by hydrodynamie injection \\
E & HFD+pGenesil-1.1 $(100 \mathrm{ug} / \mathrm{kg})$ by hydrodynamie injection \\
F & HFD+shRNA-NRP1 $(100 \mathrm{ug} / \mathrm{kg})$ by hydrodynamie injection \\
\hline
\end{tabular}

Table 2. Primer sequence of real-time PCR 


\begin{tabular}{|c|c|c|}
\hline Gene & Primer sequence $\left(5^{\prime}-3^{\prime}\right)$ & Length \\
\hline \multirow[t]{2}{*}{ TNF-a } & СССТСАСАСТCAGATСАТСТTСТ & 23 \\
\hline & GCTACGACGTGGGCTACAG & 19 \\
\hline \multirow[t]{2}{*}{ IL-1b } & GCAACTGTTCCTGAACTCAACT & 22 \\
\hline & ATCTTTTGGGGTCCGTCAACT & 21 \\
\hline \multirow[t]{2}{*}{ IL-6 } & TAGTCCTTCCTACCCCAATTTCC & 23 \\
\hline & TTGGTCCTTAGCCACTCCTTC & 21 \\
\hline \multirow[t]{2}{*}{ IL-10 } & GCTCTTACTGACTGGCATGAG & 21 \\
\hline & CGCAGCTCTAGGAGCATGTG & 20 \\
\hline \multirow[t]{2}{*}{ JNK } & AGCAGAAGCAAACGTGACAAC & 21 \\
\hline & GCTGCACACACTATTCCTTGAG & 22 \\
\hline \multirow[t]{2}{*}{$\mathrm{IKK} \beta$} & ACAGCCAGGAGATGGTACG & 19 \\
\hline & CAGGGTGACTGAGTCGAGAC & 20 \\
\hline \multirow[t]{2}{*}{ NRP-1 } & GGAAGCTCTGGGCATGGAAT & 20 \\
\hline & AGGAATCCTCTCCGGGAGTC & 20 \\
\hline \multirow[t]{2}{*}{ GAPDH } & AGGTCGGTGTGAACGGATTTG & 21 \\
\hline & TGTAGACCATGTAGTTGAGGTCA & 23 \\
\hline
\end{tabular}

Table 3. The fasting plasma glucose, serum transaminase, and lipid levels of every groups

\begin{tabular}{|lllllll|}
\hline indexes & Group A & Group B & Group C & Group D & Group E & Group F \\
\hline FPG $(\mathrm{mmol} / \mathrm{L})$ & $4.82 \pm 0.35$ & $8.60 \pm 0.45^{*}$ & $8.71 \pm 0.49 *$ & $10.93 \pm 0.86 \#$ & $8.67 \pm 0.47 *$ & $4.92 \pm 0.38$ \\
$\mathrm{HDL}(\mathrm{mmol} / \mathrm{L})$ & $1.78 \pm 0.38$ & $1.73 \pm 0.30$ & $1.65 \pm 0.35$ & $1.55 \pm 0.33$ & $1.68 \pm 0.37$ & $1.76 \pm 0.40$ \\
$\mathrm{LDL}(\mathrm{mmol} / \mathrm{L})$ & $0.67 \pm 0.14$ & $1.51 \pm 0.17 *$ & $1.52 \pm 0.21^{*}$ & $1.94 \pm 0.27 \#$ & $1.54 \pm 0.24^{*}$ & $0.73 \pm 0.18$ \\
$\mathrm{TG}(\mathrm{mmol} / \mathrm{L})$ & $1.25 \pm 0.20$ & $1.08 \pm 0.25$ & $1.11 \pm 0.28$ & $1.23 \pm 0.27$ & $1.12 \pm 0.29$ & $1.21 \pm 0.23$ \\
$\mathrm{AST}(\mathrm{IU} / \mathrm{L})$ & $13.42 \pm 1.73$ & $21.25 \pm 3.29 *$ & $21.52 \pm 3.43^{*}$ & $32.08 \pm 3.36 \#$ & $21.83 \pm 3.31^{*}$ & $13.79 \pm 1.84$ \\
$\mathrm{ALT}(\mathrm{IU} / \mathrm{L})$ & $16.75 \pm 2.16$ & $52.32 \pm 10.33^{*}$ & $51.89 \pm 10.17 *$ & $73.57 \pm 12.20 \#$ & $52.16 \pm 10.27 *$ & $17.02 \pm 2.42$ \\
\hline
\end{tabular}

FPG: fasting plasma glucose; HDL: high-density cholesterol; LDL: low-density cholesterol; TG: triglycerides; AST: aspartate aminotransferase; ALT: alanine aminotransferase. ${ }^{\#} P<0.05$ for Group D vs the other groups; ${ }^{*} P<0.05$ for Group B, C, E vs group A, F, n=6 mice/group.

\section{Figures}




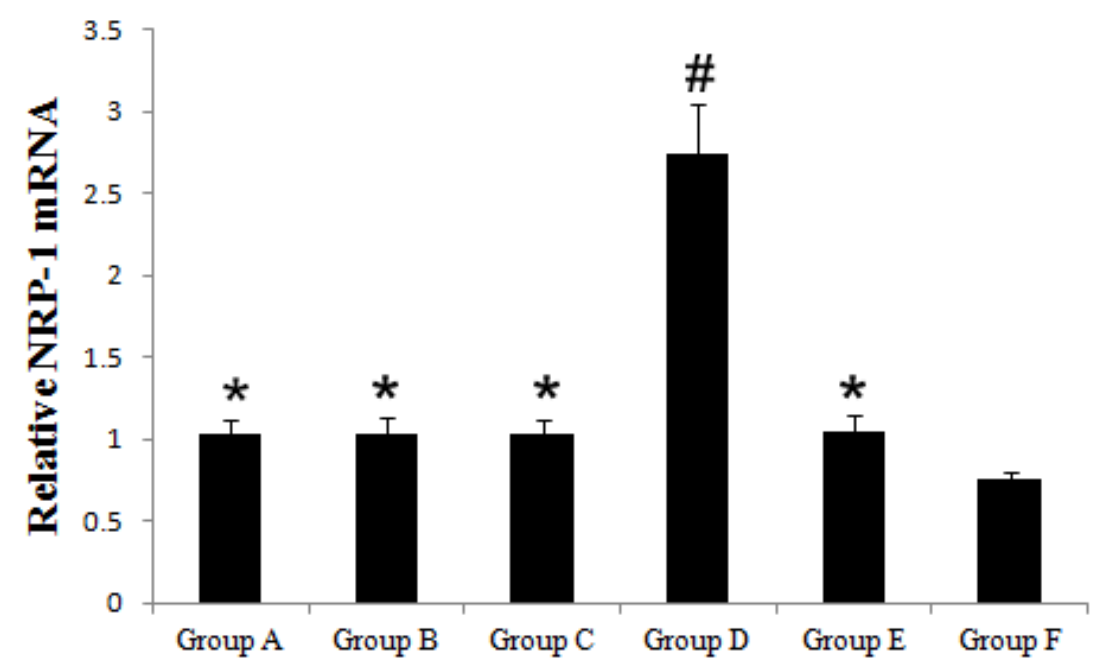

Figure 1

The relative expression of liver NRP-1 mRNA at every group $(n=6)$. Statistical differences between groups with various treatments were identified using one-way ANOVA followed by the Student-Newman-Keuls (SNK) test; \# $\mathrm{P}<0.05$ for group $\mathrm{D}$ compared with other groups; * $\mathrm{P}<0.05$ for group $\mathrm{A}, \mathrm{B}, \mathrm{C}, \mathrm{E}$ compared with group $\mathrm{F}$.
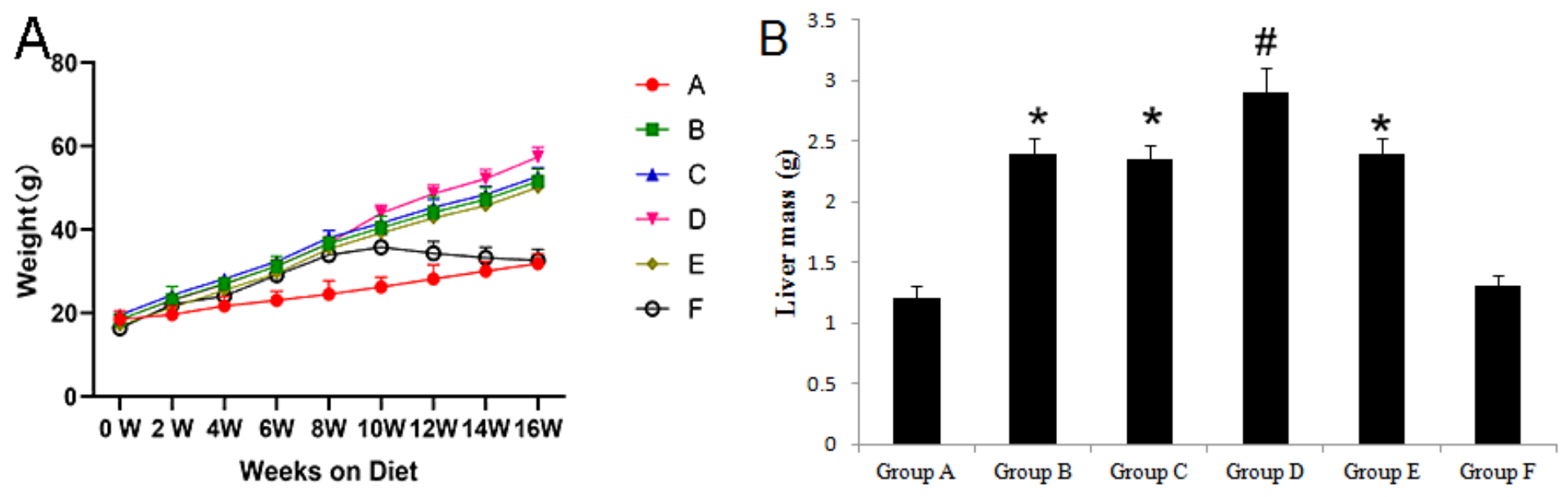

Figure 2

The relative NRP-1 protein expression of liver tissues of each group $(n=6)$. (A). A detailed comparison of various groups after treatment. Statistical differences between groups with various treatments were identified using oneway ANOVA followed by the Student-Newman-Keuls (SNK) test; \#P<0.05 for group D compared with other groups; ${ }^{*} \mathrm{P}<0.05$ for group $\mathrm{A}, \mathrm{B}, \mathrm{C}, \mathrm{E}$ compared with group $\mathrm{F}$. (B) Western blot analysis for NRP-1 protein expression of liver tissues of mouse from each group. 

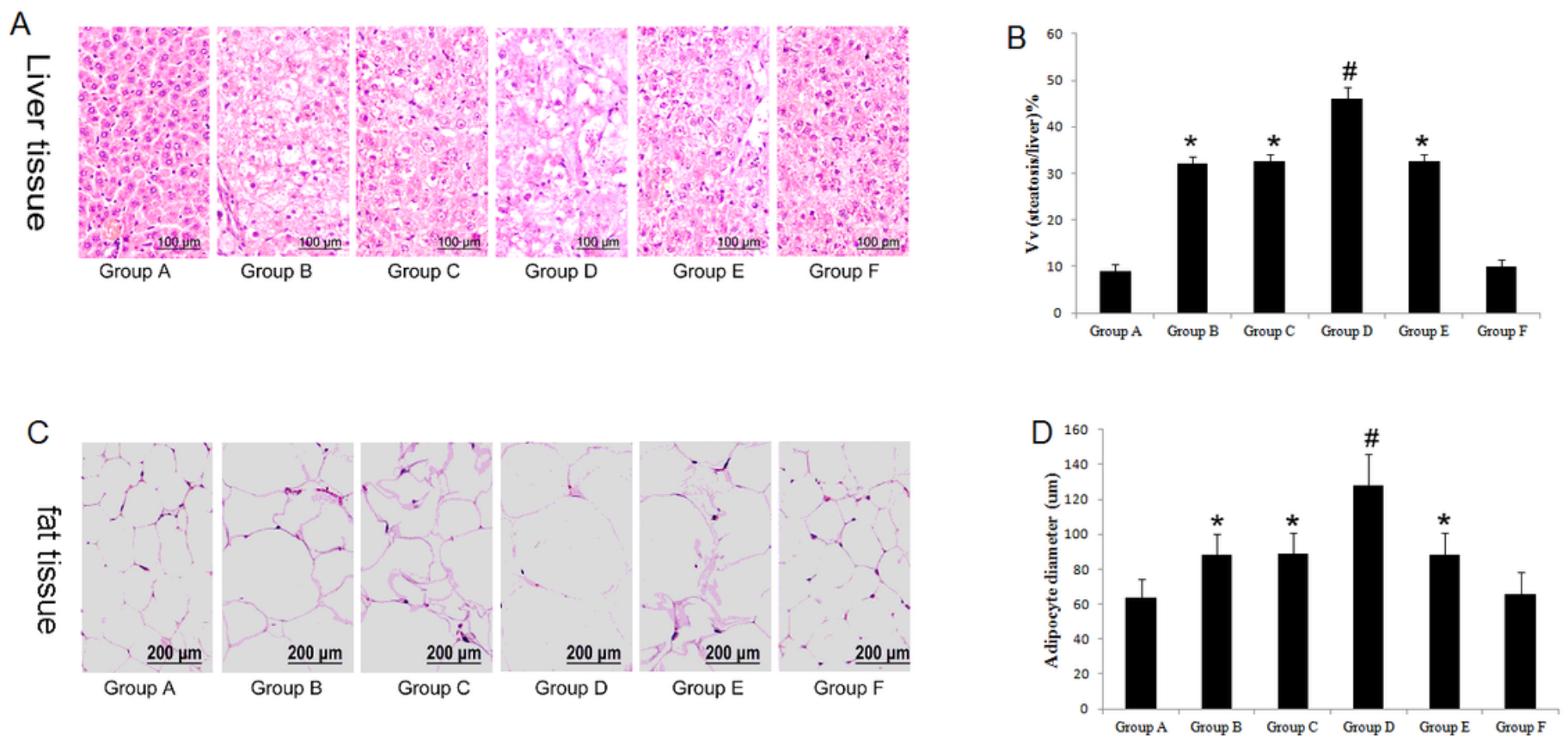

\section{Figure 3}

The body weight and liver mass of mouse in each group $(n=6)$. (A) The body weight of mouse in each group. (B) A detailed comparison of various groups after treatment. Statistical differences between groups with various treatments were identified using one-way ANOVA followed by the Student-Newman-Keuls (SNK) test; the liver mass of mouse in each group. $\# \mathrm{P}<0.05$ for group $D$ compared with other groups; $* \mathrm{P}<0.05$ for group $B, C, E$ compared with group A, F.

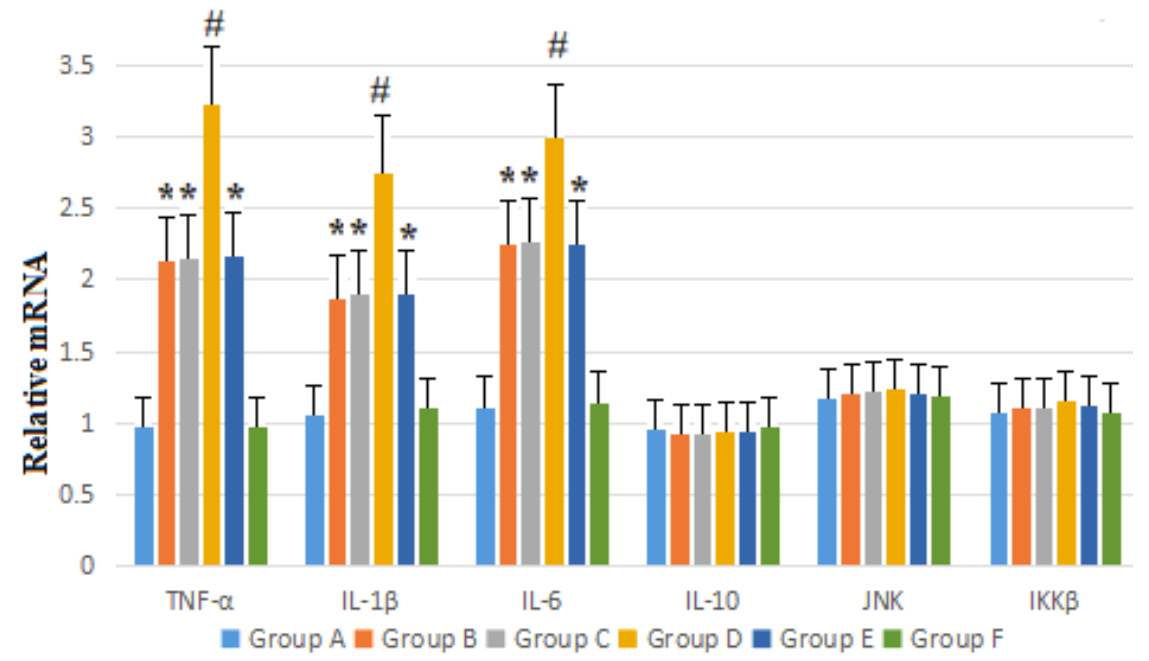

\section{Figure 4}

The histological analyses of mouse in each group $(n=6)$. (A) The histological analyses of liver steatosis of mouse in each group (H\&E stain, $100 \mu \mathrm{m}$ ). (B) Statistical differences between groups with various treatments were identified using one-way ANOVA followed by the Student-Newman-Keuls (SNK) test; the liver steatosis ratio of mouse in each group $(n=6), \# P<0.05$ for Group $D$ compared with other groups; ${ }^{*}<<0.05$ for Group $B, C, E$ compared with Group A, F. (C) The fat tissue of mouse in each group (H\&E stain, $200 \mu \mathrm{m}$ ). (D) Statistical differences between 
groups with various treatments were identified using one-way ANOVA followed by the Student-Newman-Keuls (SNK) test; the adipocyte diameter of mice at every groups $(n=6)$. \#P<0.05 for Group $D$ compared with other groups; *P $<0.05$ for Group B, C, E compared with Group A, F.

A

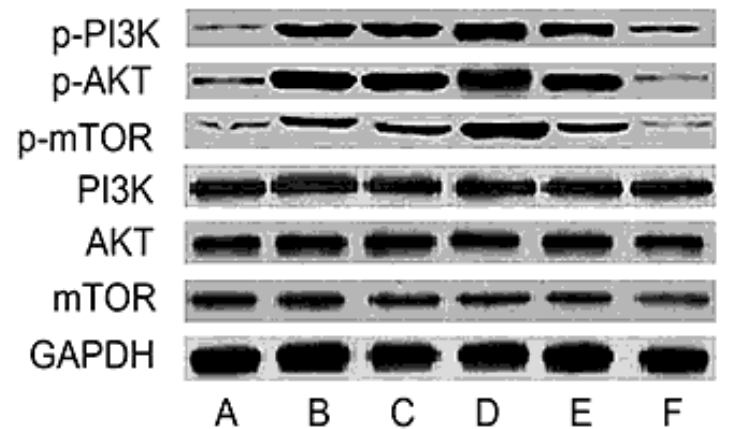

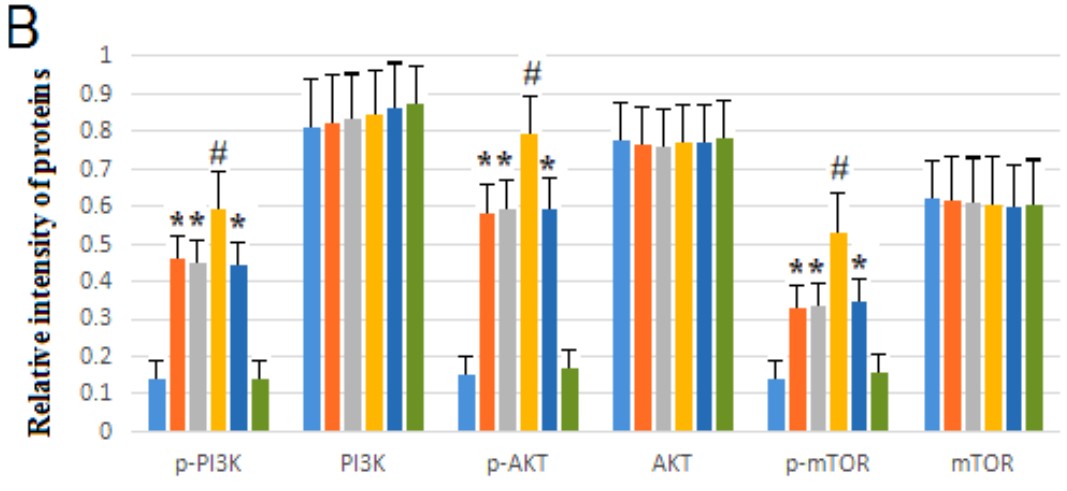

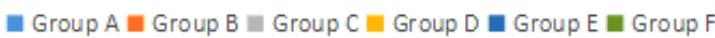

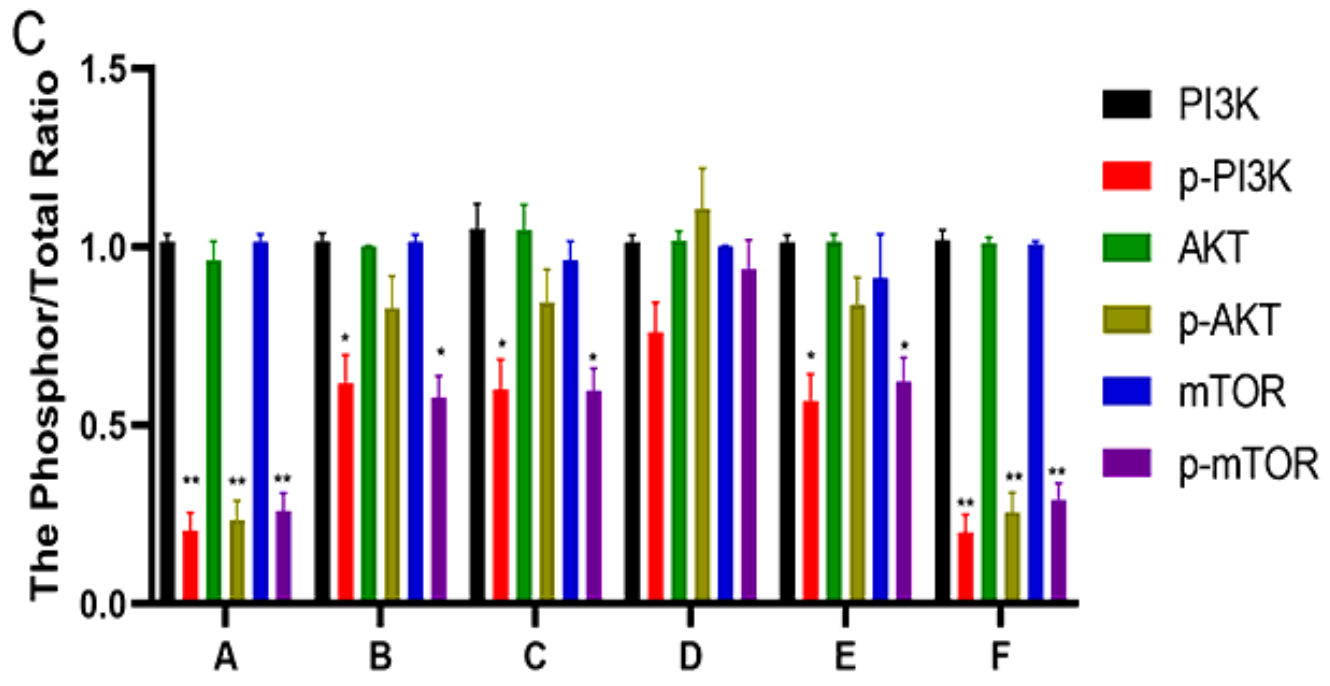

Figure 5

Quantification of pro-inflammatory cytokines. qRT-PCR was conducted in order to analyze the expression of tumor necrosis factor- $a$ (TNF-a), interleukin-1 $\beta$ (IL-1 $\beta$ ), interleukin-6 (IL-6), interleukin-10 (IL-10), c-Jun N-terminal kinases (JNK), and inhibitor of nuclear factor kappa-B kinase subunit beta(IKK- $\beta$ ). Statistical differences between groups with various treatments were identified using one-way ANOVA followed by the Student-Newman-Keuls (SNK) test; $\# \mathrm{P}<0.05$ for Group D compared with other groups; ${ }^{*}<0.05$ for Group $B, C, E$ compared with Group $A, F, n=6$ mice/ group. 

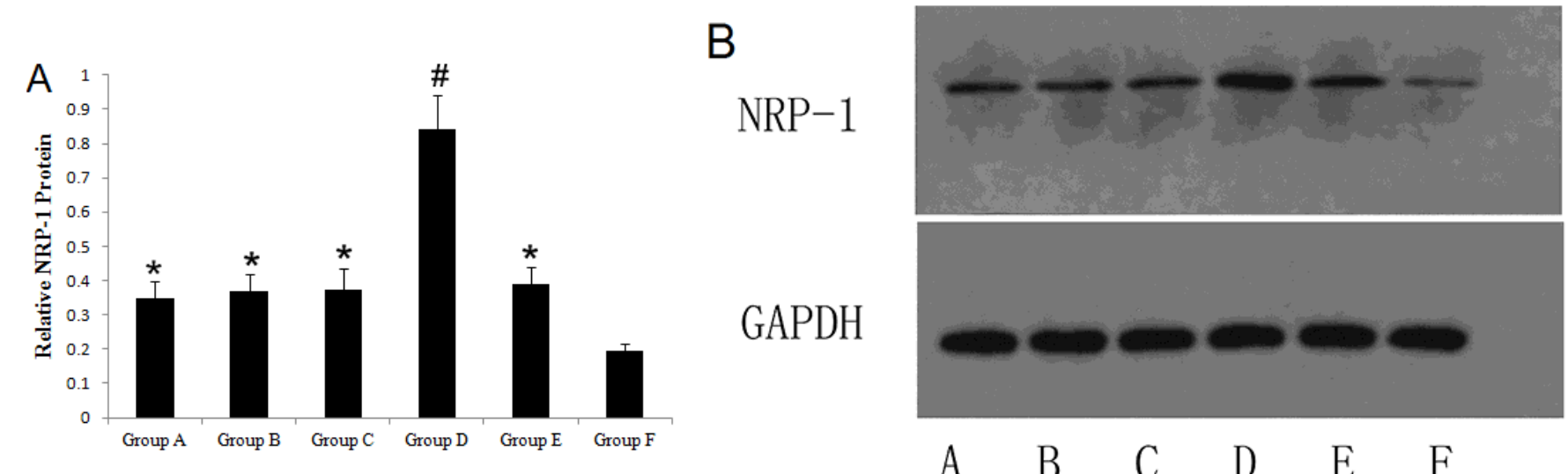

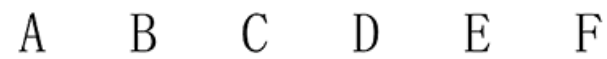

\section{Figure 6}

The related proteins expression of PI3K/AKT/mTOR signaling pathway. (A) The PI3K, AKT, mTOR, p-PI3K, p-AKT and p-mTOR proteins expression of mouse in each Group (western blot analysis). (B) The relative PI3K/GAPDH, AKT/GAPDH, mTOR/GAPDH, p-PI3K/GAPDH, p-AKT/GAPDH and p-mTOR GAPDH intensity of mouse in each group $(n=6)$. Statistical differences between groups with various treatments were identified using one-way ANOVA followed by the Student-Newman-Keuls (SNK) test; \#P<0.05 for Group D compared with the other groups; *P $<0.05$ for Group B, C, E compared with Group A, F. (C). The phosphor/total ratio of each protein. Statistical differences between groups with various treatments were identified using one-way ANOVA followed by the Student-NewmanKeuls (SNK) test; * $p<0.05,{ }^{* *} p<0.01$ compared with unphosphored protein. 